\title{
Forecasting Inflation Rate of India using Neural Networks
}

\author{
S. A. Jyothi Rani \\ Assistant Professor \\ Department of Statistics \\ Osmania University \\ Hyderabad - 500 007, India
}

\author{
V. V. Haragopal \\ Professor \\ Department of Statistics \\ Osmania University \\ Hyderabad - 500 007, India
}

\author{
M. Krishna Reddy \\ Professor \\ Department of Statistics \\ Arba Minch University \\ Arba Minch, Ethiopia
}

\begin{abstract}
In this paper, Inflation constitutes one of the major economic problems in emerging market economies that requires monetary authorities to elaborate tools and policies to prevent high volatility in prices and long periods of inflation. This paper outlines to forecast monthly inflation rate of India by using neural networks on the evaluation of set of variables. The data used for estimating the models for the period July 1994 to March 2008, for demonstration purpose of the two methodologies. The two neural network models Backpropagation neural network model or Backpropagation Network model (BPN model) and the recurrent neural network model (RNN model) under static and dynamic forecasts respectively are used in this study in forecasting inflation rate. The results of the neural network models under static and dynamic forecasts are also compared with the traditional econometric model. The results shows that the RNN model under the dynamic forecast is performing better than the BPN model under static forecast and the traditional econometric model in forecasting the inflation rate.
\end{abstract}

\section{General Terms}

Backpropagation Network model, Recurrent Neural Network, feedforward, feedback, static, dynamic, Backpropagation Algorithm, forecasting

\section{Keywords}

Neural networks, inflation, econometric model, root mean squared error (RMSE), mean absolute error (MAE), and mean absolute percentage error (MAPE).

\section{INTRODUCTION}

The term "inflation" usually refers to a measured rise in the general level of prices of goods and services in an economy over a period of time. A chief measure of price inflation is the inflation rate, which is the percentage change in a price index over time. Inflation can cause adverse effects on the economy. Inflation may widen an income gap between those with fixed incomes and those with variable income. An increase in the general level of prices implies a decrease in the purchasing power of the currency. High or unpredictable inflation rates are regarded as harmful to an overall economy they add inefficiencies in the market, and make it difficult for companies to budget or plan long-term. Inflation can impose hidden tax increases, as inflated earnings push taxpayers into higher income tax rates. A high trade deficit may lead to a fall in the value of the domestic currency and will push up the import cost, which fuels further the rising inflation. Thus controlling the inflation rate is one of the major objectives of the Governments and Central Banks all over the world and a good forecast of inflation rate is a very important of the economy. In a county like India, where almost one fourth of the population lives below the poverty line, the Government needs to have a fair idea about future movement of the of the inflation rate to take appreciate actions at the earliest to achieve the policy objectives.

In the Indian economy, studies were being made on inflation using macro-econometric models. The studies on inflation were mainly based on Keynesian approach, Monetarist approach, Structuralist approach (Paul and Bhanumurthy, 1999-2000; Krishnamurthy, 2002). As the Indian economy has a share of agriculture, the agricultural prices are more influenced by agricultural supply, due to this macro-economic models may not adequately capture the generating process for forecasting inflation rate (Barman, 2002).

For forecasting inflation rate, univariate and multivariate models also have been used widely than other traditional models. The traditional linear econometric or time series models usually have poor forecasting performance in nonlinear cases (Binner et al., 2005; Moshiri and Cameron, 1998). In other words, linear regression or time series methods many not be adequate to predict economic behavior.

Recently, artificial neural network (ANN) or simply neural network (NN) has been used as research tools in several fields. In particular, $\mathrm{NN}$ exercises a parameterized nonlinear function that can approximate nonlinear systems for prediction purpose. As far as the economic applications are concerned, NN has been widely applied to financial and macro-econometric areas (Aminian, et al., 2006; Binner, Gazely and Chen, 2002). The backpropagation neural network (BPN) model has been the most popular form of NN model used for forecasting (Mausumi et al., 1994; Hill et al., 1996). It is a feedforward neural network with a learning process in both hidden layer and output layer units. The BPN model is statistic having a learning process in both hidden and output layers in a forward direction. The recurrent neural network (RNN) allows the solution process to be dynamic. As far as the economic applications are concerned, the RNN is one of the most interesting types of NN model, as it is able to capture the dynamic behavior of the series, but RNN models have not been developed and applied as much as others in economics.

In this study, the forecasting accuracy of two models of neural networks, BPN and RNN are compared with the traditional econometric model.

A brief review of neural networks is given in Section 2 . Section 3 explains the methodology of forecasting the inflation rate using neural networks and traditional econometric model. Results and conclusions are given in Section 4. 


\section{BRIEF REVIEW OF NEURAL NETWORKS}

In modern age of forecasting, there has been a great interest in studying the NN forecasting in economics, financial, business, engineering applications including GDP growth, inflation rate, stock returns, currency in circulation, electricity demand, construction demand and exchange rates (Refenes and White, 1998; Bordoli, 2005). NN can approximate nonlinear systems for prediction purpose. Through NN one can model the relationships between one or several input (independent) variables and one or several output (dependent) variables. NN is mathematical system that is comprised of a number of processing units, also known as neurons that are linked via weighted connections. A neuron is essentially an equation, which is often referred to as transfer function. A neuron receives weighted signals from other neurons, sums them, transforms them using the transfer function and generates an output.

BPN is currently one of the most interesting NN models for forecasting (Gent and Sheppard, 1992). Because BPN model is implemented by adding learning process from hidden layer to output layer, it generally outperforms structural econometric models (Binner et al., 2005; Moshiri and Cameron, 1998; Moshiri, Cameron, and Scuse, 1999). Since BPN is a feed forward neural network, this model have the first layer, known as input layer which accepts the data and sends the weighted signal to the neurons in the second layer. The neurons in the second layer, combine the weights, generate an output by transforming the combined effect received from first layer neurons suitably and send it to the neurons in the third layer. The last layer neurons, known as the output layer generates an output. The layers between input (first) layer and output (last) layer are known as hidden layers. The number of hidden layers and the number of hidden units per hidden layer are not necessarily known and are adjusted to increase the learning complexity. Figure 1 shows the architecture of BPN model.

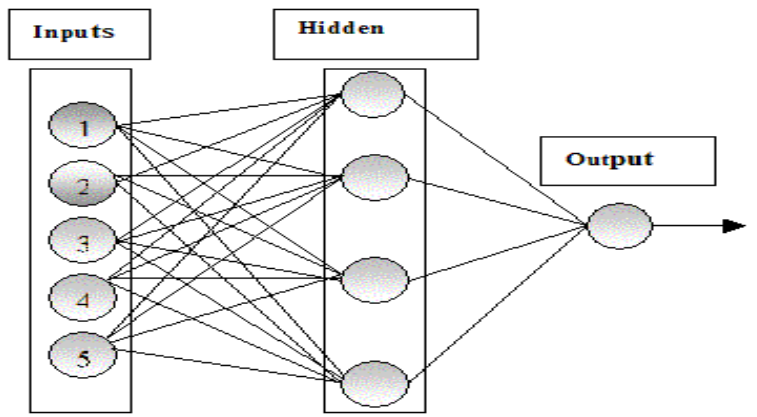

Figure 1: Architecture of BPN model with one hidden layer

For a feedforward BPN with one hidden layer (shown in Figure 1), using an input vector $X_{1}, X_{2}, \ldots ., X_{n}$, the output is obtained by using the equation,

$$
y=f\left(\sum_{h=1}^{l} w_{h o} g\left(\sum_{i=1}^{n} w_{i h} u_{i}\right)\right)
$$

where $w_{i h}$ : the weight between the $i$ th input and the $h$ th hidden node, $w_{h o}$ : the weight between the $h$ th hidden node and the output node, $\mathrm{n}$ : the number of inputs, $1:$ the number of hidden nodes, $\mathrm{f}$ : the activation function used in the output layer, $g$ : the activation function used in the hidden layer

The backpropagation (BP) algorithm is the most widely used technique for training BPN. The BP technique is an iterative gradient-descent learning algorithm in which weights in the network are iteratively modified to minimize the error between desired and actual output values. For all output units over all the input patterns, the learning process is maintained on an epoch-by-epoch basis until synaptic weights and bias level of the network stabilized. There are two alternative ways of training a network - (i). The Batch mode and (ii). The Sequential mode. This study adopted the sequential mode approach. Under this mode of BP, updating of network weights is performed after the presentation of each training example. Training of the network is being stopped when the absolute rate of change in the error per epoch is sufficiently small.

The RNN model is a dynamic network in that it has an internal feedback. Internal feedback can refer to feedback to input units from either hidden units (Elman, 1988) or from output units (Jordan, 1986). Figures 6 and 7 shows the structure of these two types of RNN model.

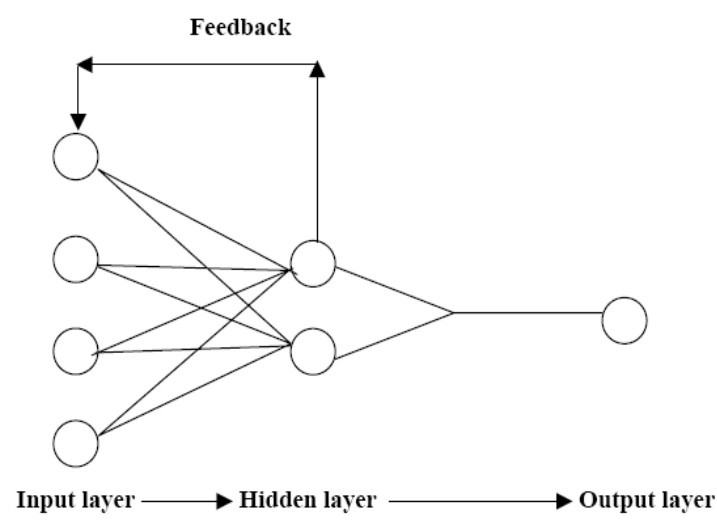

Figure 2: Architecture of RNN with feedback to input units from hidden units

Feedback

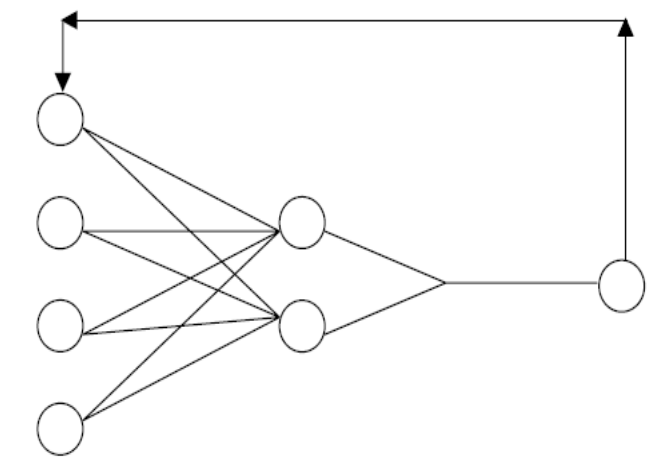

Input layer $\longrightarrow$ Hidden layer $\longrightarrow$ Output layer

Figure 3: Architecture of RNN with feedback to input units from output unit

In figure 2 , the input layer units at time $(t+1)$ receive as input not only the vectors for time $(t+1)$, but also hidden layer outputs of time $t$. The new inputs, i.e., the feed-back hidden layer outputs, are called context vectors and represent a kind of short-term memory. The initial values of the context 
vectors are set to zero and the fed-back weights connecting hidden unit outputs to context vectors are fixed at 1 . The extra weight $\delta$ that connect context vectors to the hidden units are initiated randomly and thereafter are adjusted like all other weights in the network.

In figure 3 , the input layer units at time $(t+1)$ receive as input not only the input vectors for time $(t+1)$, but also the final output vectors of time $t$. The new inputs, i.e., fed-back final output vectors are called state vectors. As in figure 2, the feedback weights connecting the final output vectors and the state vectors are fixed at 1 , and the extra weights $\delta$ that connect state vectors to the hidden units are initiated randomly and thereafter adjusted like all other weights in the network.

RNN is a dynamic network and is able to capture the dynamic behavior of complex data, especially nonlinear time series (Kuan and White, 1994). The learning algorithm in the RNN model is exactly same as that in the BPN model, using gradient descent rule, which adjusts the weights based on the derivatives of the error with respect to weights. Due to the presence of the feedback, the derivatives become more complicated and so the computations take much loner. As a result, the learning time in RNN model is usually much longer than that in BPN models.

\section{DATA AND METHODOLOGY}

\subsection{Data}

Inflation measures the change in overall level of prices in an economy. Broad measures of inflation adopted are based on the Wholesale Price Index (WPI), the Producer Price Index (PPI), the Consumer Price Index (CPI), and Gross Domestic Product (GDP) deflator. In India, officially the Wholesale Price Index (WPI) is used to measure the inflation. The coverage of the WPI is broader than the CPI and thus it is presumed that the WPI based inflation is a better measure than the CPI. The data is collected from the official website (http://www.rbi.org.in) of Reserve Bank of India (RBI). The empirical analysis uses monthly data for the period July 1994 to March 2008. The monthly data for the WPI (as base 1993$94=100$ ) is obtained as the simple average if the weekly data. The explanatory variables used for the analysis of forecasting inflation rate are: Money Supply (broad money), Industrial Production, CNX-NIFTY stock index (close value), Call Money Rates, and Exchange rate of Rupee per Dollar. The data is normalized between -1 and +1 . The figures generated here are, to explain the concept of the procedure.

\subsection{Methodology}

The are two procedures to train the neural network model in the literature. They are: (i). the entire data set is divided into two sets - 'training set' and 'testing set'. (ii). the entire data set is divided into three sets- 'training set' 'validation set' and 'test set'. In the present study, the first procedure is used by dividing the entire data into two sets. The models are estimated based on data from training set and the forecast performance is assessed based on out-of-sample forecast errors corresponding to the 'test set'. The Neurosolutions 5.1 is used to train BPN and RNN models and to generate out-ofsample forecasts. The two neural network models are training using BP algorithm. The EViews 5.1 too is used to construct the traditional model of simple econometric model i.e., multiple regression model and to generate out-of-sample forecasting which is estimated by ordinary least square method. In this study, root mean squared error (RMSE), mean absolute error (MAE), and mean absolute percentage error (MAPE) are used to evaluate the forecast efficiency of BPN, RNN, and traditional multiple regression model for its comparison.

\subsection{Forecasting Inflation Rate using BPN}

In this study, the inflation rate is forecasted depending on the different explanatory variables: Money Supply (broad money), Industrial Production, CNX-NIFTY stock index (close value), Call Money Rates, and Exchange rate of Rupee per Dollar using BPN model (static model) and is trained using BP algorithm. The Neurosolutions 5.1 is used to train BPN and to generate the out-of-sample forecasts. The following assumptions are used to train the BPN model:

Data: monthly data from July 1994 to March 2008, Network architecture: BPN, Training Algorithm: BP algorithm (gradient-descent rule), Learning rate: 0.4 , Momentum rate: 0.25 , Number of epochs: 1600 , Number of observations: 165 and Activation/ transfer function: tanh- sigmoid

\subsection{Forecasting Inflation Rate using RNN}

Under the dynamic model of RNN, the inflation is forecasted depending on the explanatory variables: Money Supply (broad money), Industrial Production, CNX-NIFTY stock index (close value), Call Money Rates, and Exchange rate of Rupee per Dollar. The BP algorithm is used to train the RNN model which is similar to training the BPN model. The Neurosolutions 5.1 is used to train RNN and to generate outof-sample forecasts with the following assumptions:

Data: monthly data from July 1994 to March 2008, Network architecture: RNN, Training Algorithm: BP algorithm (gradient-descent rule), Learning rate: 0.5 , Momentum rate: 0.3 , Number of epochs: 1850 , Number of observations: 165 and Activation/ transfer function: tanh- sigmoid.

\subsection{Forecasting Inflation Rate using Econometric Model}

The traditional multiple regression model is used to forecast the inflation rate with the explanatory variables: Money Supply (broad money), Industrial Production, CNX-NIFTY stock index (close value), Call Money Rates, and Exchange rate of Rupee per Dollar. This model is given by

$$
Y=\beta_{0}+\beta_{1} X_{1}+\beta_{2} X_{2}+\beta_{3} X_{3}+\beta_{4} X_{4}+\beta_{5} X_{5}+\epsilon
$$

For the evaluation of this model, the EViews 5.1 is used to forecast the inflation rate with ordinary least squares method.

\section{RESULTS AND CONCLUSION 4.1 Results}

The two neural network models BPN (static model) and RNN (dynamic model) are used to forecast the monthly inflation rate of India, and the results obtained of the neural network models are compared with the results of traditional econometric model. The forecasting accuracy of these three models is tested by using RMSE, MAE, and MAPE values.

In BPN and RNN models, $80 \%$ of the data is used as training set to estimate the models and the remaining $20 \%$ of the data is used as testing set for assessing the forecast performance based on out-of-sample forecasts. The results obtained by neural network and traditional models are shown in Table 1 as follows: 
Table 1: Results obtained by BPN, RNN, and traditional model

\begin{tabular}{|c|c|c|c|c|}
\hline Model & $\begin{array}{c}\text { No. of } \\
\text { observations }\end{array}$ & RMSE & MAE & MAPE \\
\hline BPN & 165 & 0.911 & 0.622 & 0.742 \\
\hline RNN & 165 & 0.487 & 0.224 & 0.129 \\
\hline $\begin{array}{c}\text { simple } \\
\text { econometric } \\
\text { model }\end{array}$ & 165 & 1.847 & 2.312 & 1.261 \\
\hline
\end{tabular}

\subsection{Conclusions}

Empirical evaluation shows that RNN model is performing better than BPN and traditional models to forecast inflation rate of India. Also, the performance of BPN is better than the traditional econometric model. It shows that neural network models are very successful in predicting nonlinear relationships. For the application studied in this paper, it has concluded that the RNN model (dynamic model) is significantly more accurate than the BPN model (static model) and the traditional model. Since the RNN has the internal feedback which allows it to capture the dynamic structure of the data, it has able to outperform the BPN model and the traditional model. As most of the economic series are dynamic, therefore the RNN model can be a good alternative to the time series and economic series data.

Though, the above procedures show good performance, one can try the data sets considered here be evaluated by $\mathrm{ARCH}$ and GARCH models for better model fit for the future study.

\section{REFERENCES}

[1] Aminian, F., Suarex, E.D., Aminian, M., and Walz, D.T. 2006. Forecasting Economic data with Neural Networks, Computational Economics, 28, 71-88.

[2] Barman, R.B. 2002. Forecasts of Economic indicators for monetary policy in India: An Assessment, Irving Fisher Committee Bulletic, 13, 80-93.

[3] Binner, J.M., Gazely, A.M., Chen, S. 2002. Financial Innovation and Divisia Money in Taiwan: A neural network approach, The European Journal of Finance, 8, 238-247.
[4] Binner, J.M., Bissoondeeal, R.K., Elger, T., Gazely, A.M., and Mullineux, A.W. 2005. A comparison of Linear Forecasting Models and Neural Networks: An Application to Euro Inflation and Euro Divisia, Applied Economics, 37,665-680.

[5] Bordoli, S. 2005. Neural Network Forecasting of Headline inflation Rate in India, Statistics and Applications, 3(1), New Series, 65-85.

[6] Gent, C.R. and Sheppard, C.P. 1992, Predicting Time Series by a Fully Connected Neural Network Trained by Backpropagation, Computing \& Control Engineering Journal, 3, 109-112.

[7] Elman, J.L.,1988. Finding Structure in Time, CRL Report 8801, Centre for Research in Language, UC San Diego.

[8] Hill, Tim, O'Connor, Marcus and Rmus, William. 1996. Neural network models for time series forecasts, Management Science, 42(7).

[9] Jordan, M.T. 1986. Serial Order: a parallel distributed processing approach, UC San Diego, Institute for Cognitive Science, Report 8604.

[10] Krishnamurty, K. 2002. Macroeconometric Models for India: Past, Present and Prospects. Economic Political Weekly, XXXV (42), 4295-4308.

[11] Kuan, C. and White, H. 1994. Artificial neural networks: an econometric perspective, Econometric Review, 13(Nov.).

[12] Mausumi, E., Khotanad, A. and Abaye, A. 1994. Artificial neural networks for some macroeconomic series: a first report, Econometric Reviews, 13(1).

[13] Moshiri, S., Cameron, N.C. 1998. Neural network versus econometric models in forecasting inflation, Journal of Forecasting, 19(3), 201-217.

[14] Moshiri, S., Cameron, N. and Scuse, D. 1999. Statisti, Dynamic and Hybrid Neural Networks in Forecasting Inflation, Computational Economics, 14, 219-235.

[15] Paul, M.T. and Bhanumurthy, N.R. 1999-2000. Money Supply, Output and Price Level Relationship in India, The Indian Economic Journal, 47(1), 42-55.

[16] Refenes, A.P. and White, H. 1998. Neural Networks and Financial Economics, International Journal of Forecasting. 